\title{
The Aachen Muon Detector for testing the local production of Scintillating Surface Detectors for AugerPrime
}

\author{
T. Bretz*, P. Ferreira, A. Garcia, T. Hebbeker, J. Kemp, C. Peters and J. Schumacher \\ Physics Institute III A \\ RWTH Aachen University \\ Aachen, Germany \\ E-mail: tbretz@physik.rwth-aachen.de
}

\begin{abstract}
The prototype of the Aachen Muon Detector was designed as an alternative for the upcoming upgrade (AugerPrime) of Scintillating Surface Detectors (SSD) for the Pierre Auger Observatory. Due to the successful application of semi-conductor photo sensors (SiPM), two portable detectors with a slightly modified design were built. They are currently applied for testing the local production of SSD detectors.

Each of the two detectors consists of eight $30 \times 30 \times 0.5 \mathrm{~cm}^{3}$ scintillator tiles. The light of each tile is collected by a dedicated wavelength-shifting fiber embedded into the tile and coupled directly to a $1.3 \times 1.3 \mathrm{~mm}^{2}$ SiPM. Reading out each channel individually with an EASIROC data acquisition chip provides spatial resolution, muon counting and an amplitude measurement for bright events. The obtained spectrum of minimum ionizing particles is nicely modulated with the single-photon equivalent (p.e.) resolution of the SiPM allowing for a very precise calibration of the system.

The use of SiPM provides a very robust solution for a lightweight and portable detector easily applicable to a wide variety of setups.
\end{abstract}

36th International Cosmic Ray Conference -ICRC2019-

July 24th - August 1st, 2019

Madison, WI, U.S.A.

\footnotetext{
*Speaker.
} 


\section{Introduction}

In the past decade, semi-conductor based photon sensors (SiPMs) have found their way into astroparticle physics. For example, the First G-APD Cherenkov Telescope has established their application successfully in imaging air-Cherenkov astronomy [1]. Although, their main limitation is still their small geometrical size of typically less than $36 \mathrm{~mm}^{3}$, they provide a simplified operation without the risk of aging due to bright light.

Their excellent single-photon resolution at high photo detection efficiency allows for an easy and accurate calibration, e.g. [2]. For the detection of low light-levels, like in the application of scintillators, this allows to obtain the calibration spectrum induced by minimum ionizing particles (MIPs) with single photon resolution, c.f. [3, 4]. Simultaneously, a high dynamic range can be achieved, c.f. [5, 6].

Scintillators are applied in astroparticle physics for example for the detection of extensive airshowers. An example is the on-going upgrade of the Pierre Auger Observatory (AugerPrime, [7]). The Pierre Auger Observatory observes ultra-high energy cosmic rays through the measurement of secondary particle cascades introduced in the Earth's atmosphere. By the measurement of the properties of these extensive air-showers, the properties of the primary particles can be deduced. For the measurement of the arrival time of the shower front and for the purpose of particle counting, 1660 water-Cherenkov counters serve as particle detectors [8]. The observatory is currently undergoing a major upgrade including an upgrade of its ground array [9]. By the application of additional dedicated photo multiplier tubes (PMTs), the dynamic range of the detectors is increased for a more precise measurement of the most energetic showers with energies around $10^{20} \mathrm{eV}$ without saturation of the data acquisition taking place. An additional, scintillator detector on top of each water-Cherenkov station complements the existing measurement. Discriminative response of both detector types promises to disentangle the electromagnetic component in the observed air showers from the muonic component providing event-wise sensitivity for the mass of the primary particle. As a prototype application, an optical module was designed and built applying SiPMs as photo sensors. Several of them are now installed in the field providing first data [6].

As an alternative solution for applied scintillator surface detector (SSD), the Aachen Muon Detector (AMD) has been designed [3]. Its concept, the design of a prototype and a recent application is discussed in the follwing.

\section{The Aachen Muon Detector (AMD)}

Instead of replacing photo multiplier tubes in an existing design with SiPMs, the concept of the Aachen Muon detector was a design dedicated for the application of SiPMs. In the original design, fluorescence light emitted by particles going through $6430 \times 30 \times 0.5 \mathrm{~cm}^{3}$ tiles of plastic scintillators was collected by wavelength shifting fibers and transmitted via clear optical fibers to $1 \times 1 \mathrm{~mm}^{2}$ SiPMs each. This concept makes use of the small size of SiPMs and provides additional spatial resolution for the through-going particles. Decreasing the effective area of the individual detectors allows for direct particle counting within a large dynamic range. This lowers the system- 
atic errors usually connected with the conversion of combined analog signal into a particle count through means of calibration.

For the application as part of the upgrade of the Pierre Auger Observatory, the detector was designed as a real muon detector installed underneath the water-Cherenkov detectors using the huge water reservoir as almost perfect shielding for the electromagnetic component of the shower. Although, the concept has proven its advantages [10], the installation below the water-Cherenkov counters and the associated emptying, lifting and refilling of the water-reservoir imposes a too high risk on the integrity of the well-working stations. Therefore, the application of a low-risk solution was decided.

\section{The MiniAMD detector}

As the original steal housing of the AMD concept was far to heavy for easy handling in a prototype detector, the implementation of a more light weight and more flexible solution based on the same detector elements was decided. Instead of 64 tiles, only 8 tiles were manufactured and mounted into a light weight aluminum frame. In several laboratory tests, the optical coupling of photons from the wavelength-shifting fibre into the clear optical fibre has shown to be delicate. To avoid this generally unnecessary coupling, in addition, the SiPMs were directly attached to the outlet of the wavelength shifting fibre from the scintillator tile. As gluing the fibre into a pre-milled groove turned out to be a tedious and time consuming process, fibers instead were placed into a fitting. Laboratory measurements showed no evidence for significant light-loss due to the worsened optical coupling from the tile into the fibre. It turned out that anticipated coating with a reflective paint at the open end of the fibre, to keep collected photons inside the fibre, was inefficient. This was solved by the gluing of a small piece of reflective foil instead. Wrapping tests during which different reflective packagings for the tiles have been compared showed the best performance if the tile was wrapped in Teflon tape. Measurements showed an average light output of 24 photon equivalents for a minimum ionizing particle going through the tile. The homogeneity of the response over the surface of the tile was characterized in a former muon tracking detector of the KASCADE experiment [11]. The measured homogeneity was consistent with the resolution of the tracking detector and the expectations from simulations. Despite the asymmetric layout of the fibre in the tile, no significant inhomogeneity was observed. For coincidence measurements, two of these dectors were built.

A picture of one open detector is shown in Fig. 1. Visible are the wrappings of the eight individual tiles and the cables from the eight corresponding SiPMs to the electrical connectors at which the bias power supply and data acquisition system is connected. A picture of a single tile without wrapping is presented in Fig. 2 together with a sketch of the design of the tile with wavelength-shifting fibre and attached SiPM.

\section{The prototype test setup}

The Physics Institute III A of RWTH Aachen University is involved in the production of the Scintillator Surface Detector (SSD) [12, 7] of the Pierre Auger Observatory. The quality of the production has to be verified for each detector individually before shipment to Argentina. An SSD 


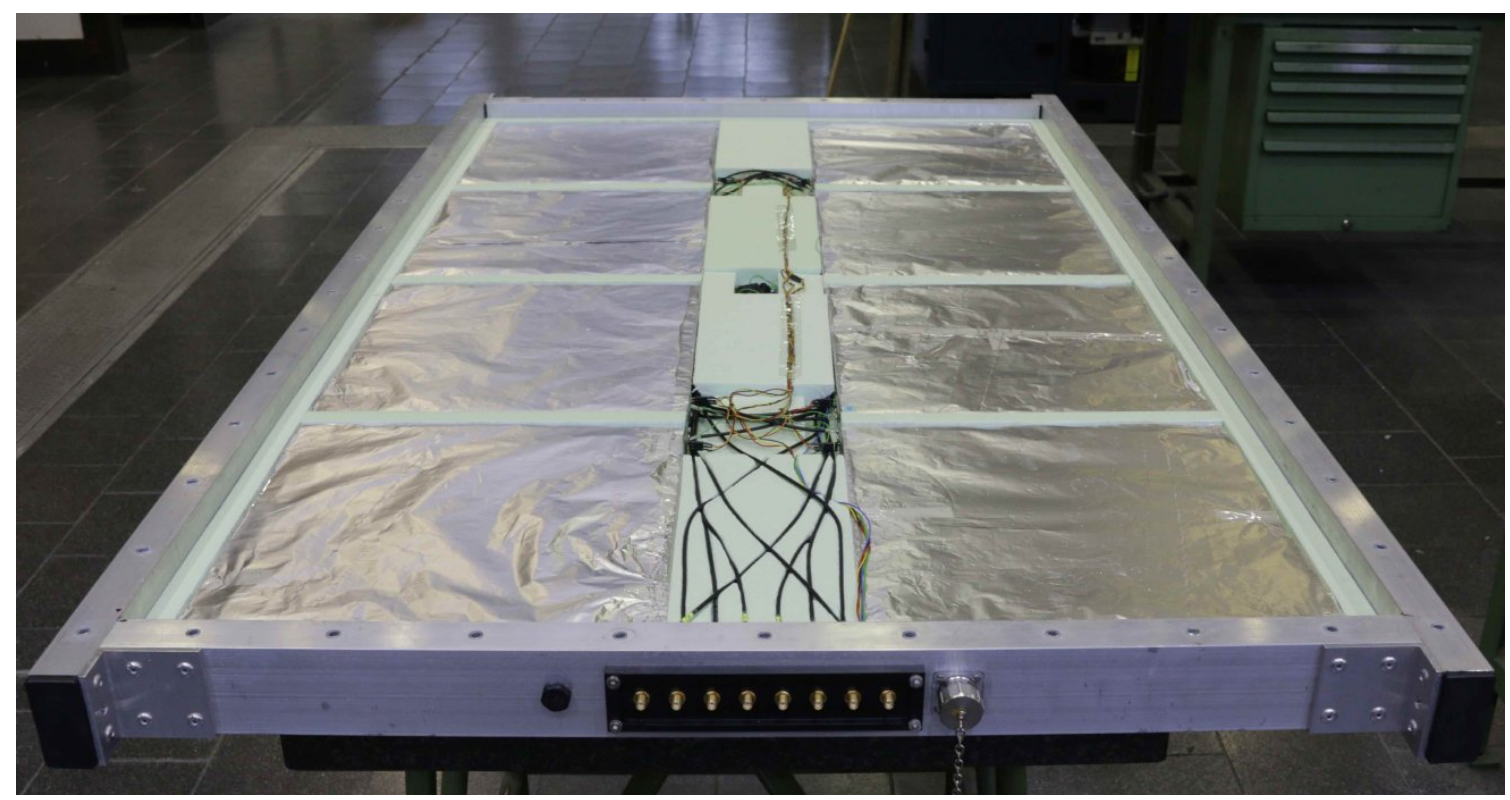

Figure 1: A picture of the MiniAMD detector. The detector's aluminum frame is visible withthe top cover removed. The eight visible individual squared are the eight individual tiles wrapped with Teflon tape and protected with an aluminum cover. The black cables connect the SiPMs attached to each tile electrically with the connectors at the front plate at which the bias supply and data acquisition system is connected.
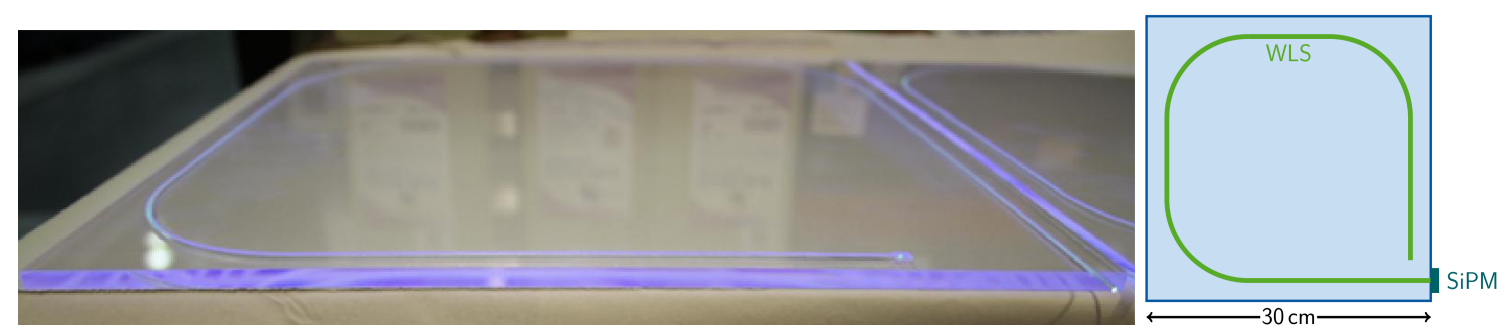

Figure 2: Visible is a plastic scintillator tile of dimensions $30 \times 30 \times 0.5 \mathrm{~cm}^{3}$ with a wavelength shifting fiber routed inside (left) and a sketch of the design of the tile (right) with the wavelength shifting fibre and the SiPM attached to it.

hosts 48 scintillator bars with a size of $160 \times 5 \times 1 \mathrm{~cm}^{3}$ each. They are arranged in two detector halves with 24 scintillator bars each, resulting in a width of the effective area of about $120 \mathrm{~cm}$. The light is collected by wavelength shifting fibers and guided onto one centrally placed PMT.

MiniAMD being a small and lightweight detector makes it an ideal trigger device for testing other detectors. It can easily be brought at the right place for triggering on cosmic muons. MiniAMD has a total length of about $140 \mathrm{~cm}$, compared to the width of the effective area of the SSDs of $120 \mathrm{~cm}$. Thus, MiniAMD is the ideal device to provide a trigger in a test stand of the SSDs. As the scintillator bars of the SSD are arranged in two separate detector halves, both halves have to be measured separately. A movable muon trigger is thus advantageous. A sketch and a picture of the developed test stand can be seen in figures 3 and 4 .

Two MiniAMD detectors are placed on a movable forklift together with their data acquisition system as well as the power supply. They have a vertical distance of $1 \mathrm{~m}$. Together with an edge 


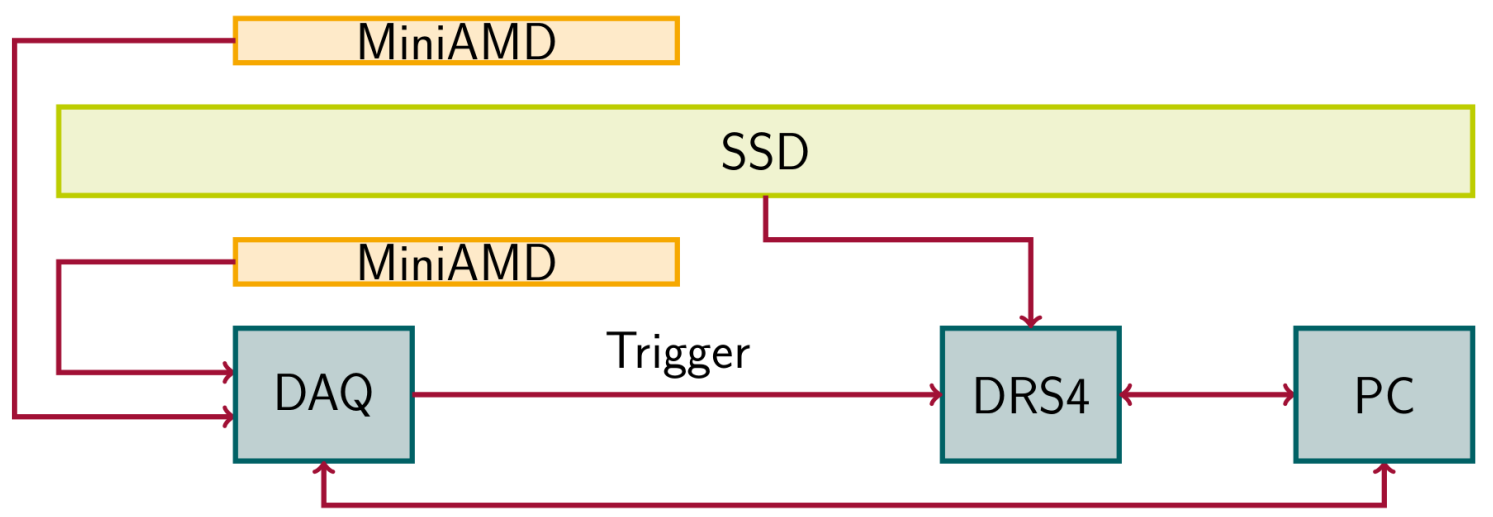

Figure 3: A sketch of the test stand to validate the local production of SSDs.

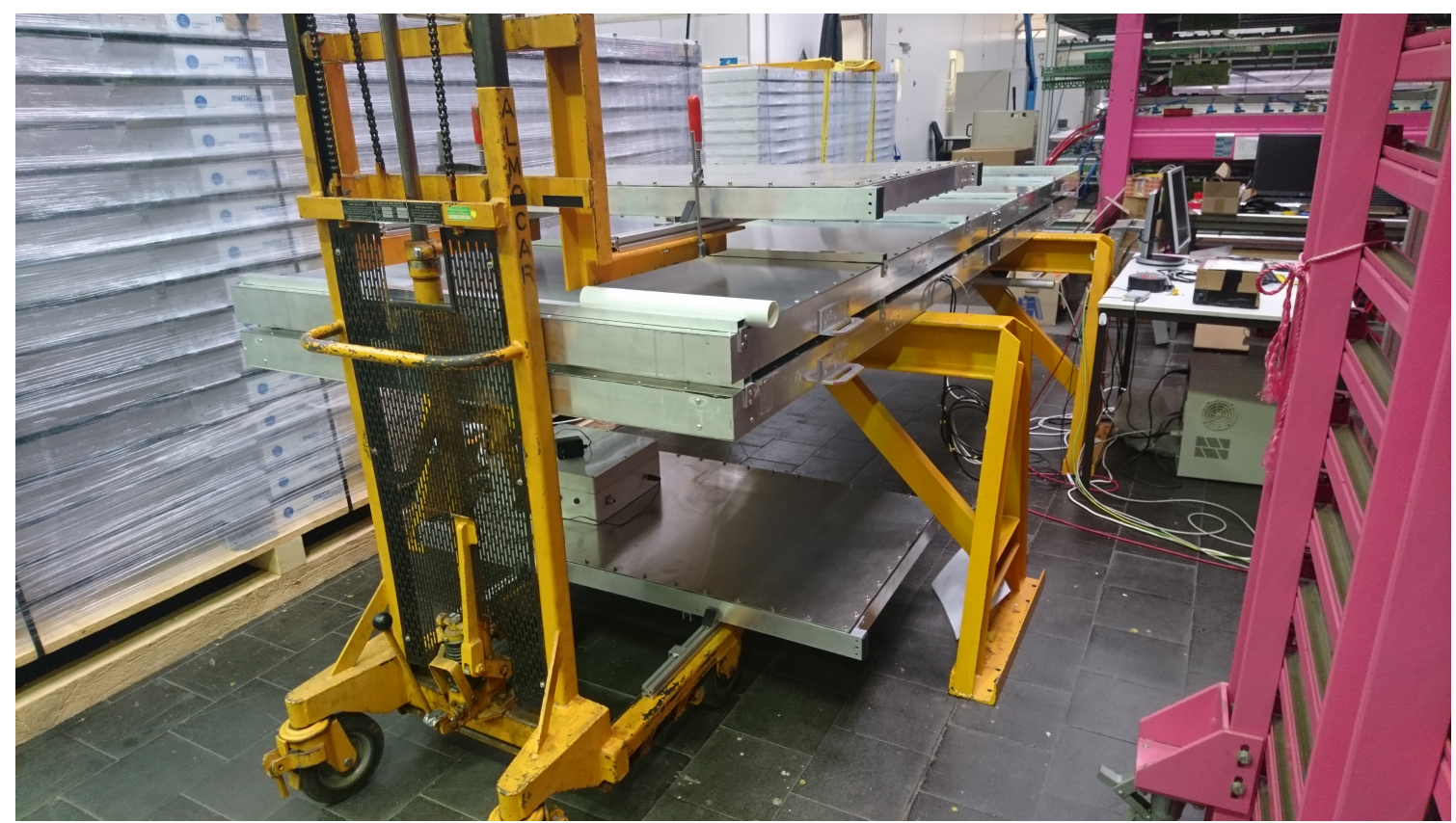

Figure 4: The test stand for the validation of the local production of SSDs. Two MiniAMD modules are mounted on a movable forklift. The MiniAMD data acquisition and power supply are placed on top of the bottom MiniAMD. On the picture, two SSDs are placed between the MiniAMD modules for testing.

length of $30 \mathrm{~cm}$ of a tile, this results in a maximum zenith angle for cosmic muons of $22^{\circ}$. The main goal of the tests is to determine the light yield of the SSD for through going muons as well as to confirm the light tightness of the detector.

The signal of the SSD PMT is recorded using a DRS4 evaluation board ${ }^{1}$. MiniAMD provides the trigger for the read out when a muon signal is detected at two tiles that are directly above each other to only allow for close to vertical muons. Due to the high analogue bandwidth of the DRS4 evaluation board of $700 \mathrm{MHz}$ and the very fast pulses of the SSD PMT, the readout is performed with $3 \mathrm{GS} / \mathrm{s}$.

\footnotetext{
${ }^{1}$ https://www.psi.ch/en/drs
} 
In addition to the muon triggered traces, also a fixed rate trigger is applied. The traces recorded with this trigger allow searching for single photo electron (p.e.) pulses of the SSD PMT. In case of a light leak of the detector box, the single p.e. rate will increase.

\section{Results}

For the muon triggered traces, a pulse finding algorithm is applied to the recorded SSD PMT traces. All found pulses are integrated and the baseline is subtracted. The resulting charges are filled into a histogram. An example is shown in figure 5. The peak created by through going

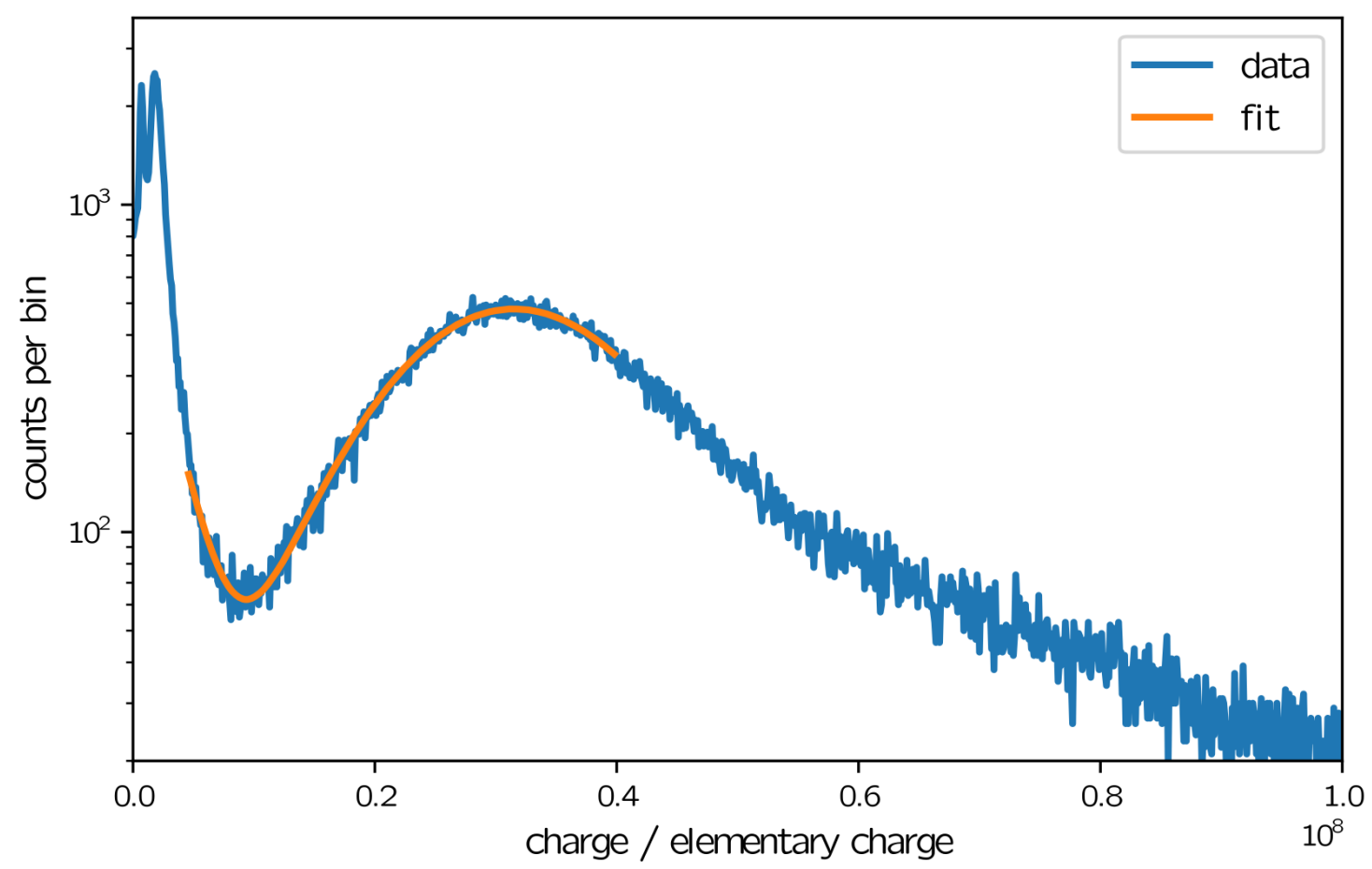

Figure 5: An example charge histogram for the muon triggered traces. The performed fit consits of an exponential to describe the pedestal and Landau distribution convolved with a Gaussian to describe the muon peak.

muons is well visible and separated from the noise pedestal. The good visibility of the moun peak proves the good performance of two MiniAMDs operated in coincidence to serve as a muon trigger. The noise pedestal results either from pulses found in the trace outside the trigger window or from muons crossing both MiniAMD detectors but not the SSD effective area. This can happen due to the slightly larger length of MiniAMD compared to the width of the SSD.

The performed fit allows to determine the position of the maximum of the muon peak as well as its width. Due to the very clean muon signal, this is done with great precision. 


\section{Conclusion}

A test stand for validation of the local production of the SSDs for the Pierre Auger Observatory has been establised based on MiniAMD as a trigger on cosmic muons. So far, 106 detectors have been tested within about one year of operation of the test stand. During this long running procedure, MiniAMD has proven its performance and reliability also over long time ranges.

\section{References}

[1] H. Anderhub et al. (FACT Collaboration), Design and Operation of FACT - The First G-APD Cherenkov Telescope, JINST 8 (2013) P06008 [doi:10.1088/1748-0221/8/06/P06008]

[2] A. Biland et al. (FACT Collaboration), Calibration and performance of the photon sensor response of FACT - the first G-APD Cherenkov Telescope, JINST 9 (2014) P10012 [doi:10.1088/1748-0221/9/10/P10012]

[3] C. Peters et al., The muon detector prototype AMD for the determination of the muon content in UHECRs, In Proc. of the 34th ICRC [PoS(ICRC2015)596]

[4] C. Peters et al., Prospects of Silicon Photomultipliers for Ground-Based Cosmic Ray Experiments, In Proc. of UHECR2016: JPS Conf. Proc. 19 (2018) 011030 [doi:10.7566/JPSCP.19.011030]

[5] T. Bretz et al., An integrated general purpose SiPM based optical module with a high dynamic range, JINST 13 (2018) P06001 [doi:10.1088/1748-0221/13/06/P06001]

[6] J. Kemp et al., Air Shower Measurement with a SiPM based Scintillator Detector, These proceedings.

[7] R. Smida et al. (Pierre Auger Collaboration), Scintillator detectors of AugerPrime Scintillator detectors of AugerPrime [PoS(ICRC2017)390]

[8] I. Alekotte et al. (Pierre Auger Collaboration), The surface detector system of the Pierre Auger Observatory, Nucl.Instr. and Meth. A 586 (2008) 3 [doi:10.1016/j.nima.2007.12.016]

[9] A. Aab et al. (Pierre Auger Collaboration), The Pierre Auger Observatory Upgrade - Preliminary Design Report [arXiv:1604.03637]

[10] C. Peters, Development of scintillator detectors for measuring muons and air showers, $\mathrm{PhD}$ Thesis (2019), RWTH Aachen University [doi:10.18154/RWTH-2019-05267]

[11] W. Doll et al., Muon tracking detector for the air shower experiment KASCADE, Nucl.Instr. and Meth. A 488 (2002) 517 [doi:10.1016/S0168-9002(02)00560-0]

[12] J. Pekala et al. (Pierre Auger Collaboration), Production and Quality Control of the Scintillator Surface Detector for the AugerPrime Upgrade of the Pierre Auger Observatory, [PoS(ICRC2017)380] 